Physics

Physics Research Publications

\title{
Magnetic properties of the superconducting state of iron-based superconductors
}
K. J. Seo
C. Fang

B. A. Bernevig

J. P. $\mathrm{Hu}$ 


\title{
Magnetic properties of the superconducting state of iron-based superconductors
}

\author{
Kangjun Seo, ${ }^{1}$ Chen Fang, ${ }^{1}$ B. Andrei Bernevig, ${ }^{2}$ and Jiangping $\mathrm{Hu}^{1}$ \\ ${ }^{1}$ Department of Physics, Purdue University, West Lafayette, Indiana 47907, USA \\ ${ }^{2}$ Princeton Center for Theoretical Science, Princeton University, Princeton, New Jersey 08544, USA
}

(Received 13 March 2009; published 19 June 2009)

\begin{abstract}
We show that features of the dynamical spin susceptibility can unambiguously distinguish between different pairing symmetries of the superconducting state in iron-based superconductors. A magnetic resonance is a

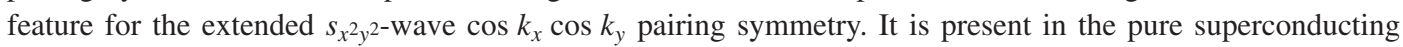
(SC) state but weakens in the mixed SC and magnetically ordered state. We calculate the random-phase approximation correction to the NMR spin-relaxation rate $1 / T_{1}$ and the Knight shift in the above states and show a good agreement with experimental results. Moreover, we argue that the energy dispersion of the magnetic resonance along the $c$ axis observed in neutron scattering experiments is also an indirect evidence supporting the $s_{x^{2} y^{2}}$ pairing symmetry.
\end{abstract}

DOI: $10.1103 /$ PhysRevB.79.235207

PACS number(s): 74.25.Ha, 74.20.Rp, 76.60.-k

\section{INTRODUCTION}

The recently discovered iron-based high-temperature superconductors ${ }^{1-5}$ have very intriguing magnetic properties ${ }^{6}$ which may hold the key to understanding their superconducting (SC) pairing mechanism. Theoretically, many possible gap pairing symmetries have been proposed. Due to the proximity of the superconducting state to a collinear antiferromagnetic state, as well as due to relatively weak phonon interactions, a magnetism-based superconducting mechanism is favored. Falsifying or verifying the prediction of pairing symmetry will be a significant step in understanding the nature of the magnetically driven (SC) pairing mechanism. Both weak and strong coupling approaches suggest that an extended $s$-wave pairing symmetry is favored..$^{7-9}$ Based on a weak-coupling approach, an $s$-wave (so called $s^{ \pm}$) state, ${ }^{8}$ in which the sign of order parameters changes between hole and electron pockets, is argued to be favored for repulsive interband interactions. However, the weak-coupling approach does not specify the exact form of order parameters and is dependent on the degree of nesting between the electron and hole pockets. In the strong coupling approach, in a recent paper, ${ }^{7}$ we showed that the pairing symmetry is determined mainly by the next-nearest-neighbor antiferromagnetic exchange coupling $J_{2}$ (Refs. 10-14) and has an explicit form in momentum space, $\cos \left(k_{x}\right) \cos \left(k_{y}\right)$. This result is model independent as long as the dominating interaction is nextnearest-neighbor $J_{2}$ and the Fermi surfaces (FSs) are located close to the $\Gamma$ and $M$ points in the Brillouin zone (BZ). The $\cos \left(k_{x}\right) \cos \left(k_{y}\right)$ changes sign between the electron and hole pockets in the Brillouin zone. In this sense, it resembles the order parameter, $s^{ \pm}$, proposed through weak-coupling general arguments. ${ }^{8,15}$

The magnitudes of superconducting gaps measured by angle-resolved photoemission spectroscopy (ARPES) at different Fermi surfaces are in a good agreement with the simple $\left|\cos \left(k_{x}\right) \cos \left(k_{y}\right)\right|$ form, ${ }^{16-18}$ but directly probing the sign change from electron to hole pockets still remains a big experimental challenge despite several theoretical proposals. ${ }^{19-23}$ Fortunately, the magnetic properties in the superconducting state may provide us with indirect evidence of the pairing symmetry. It has been pointed out that, in the extended $s$-wave SC states, ${ }^{24,25}$ there is a strong coherent peak in the dynamic spin susceptibility $\chi(Q, \omega)$ at an energy $\hbar \omega$ below the two-gap value and at a wave vector $Q$ $=(0, \pi)$ identical to the magnetic ordering wave vector in parent compounds. Recent neutron scattering experiments ${ }^{26-28}$ have observed a magnetic resonance in the superconducting states similar to the magnetic resonance observed in cuprates. This result provides an indirect evidence of the extended $s$-wave pairing if the coherent peak is a unique feature of this pairing symmetry. Neutron scattering experiments ${ }^{28}$ have also observed a significant dispersion of resonance peak along $c$ axis.

Complementing neutron scattering, NMR is an important probe of magnetic properties. The experimental results of the NMR spin-relaxation rate $1 / T_{1}$ in these superconductors have been a challenge for theories predicting the extended $s$-wave pairing symmetry due to the absence of coherent peak and the near cubic power-law dependence on temperature. ${ }^{29-32}$ Several works ${ }^{33-35}$ have addressed this problem and pointed out that for the $\cos \left(k_{x}\right) \cos \left(k_{y}\right)$ order parameter, the interband contribution to the NMR spinrelaxation rate does not exhibit a coherence peak. As such, the experimental result is not inconsistent with the pairing symmetry if the interband contribution is much larger than the intraband contribution or if the samples are strongly disordered. However, in a simple mean-field state, the intraband contribution is always larger or comparable to the interband contribution and the spin-relaxation rate still exhibits an enhancement right below $T_{c}$ owing to its fully gapped $s$-wave nature. ${ }^{33}$

In this paper, we perform a detailed calculation of the magnetic response in the superconducting state of iron-based superconductors. Within a two-orbital model and within the random-phase approximation (RPA) we address several experimentally related issues. (1) We demonstrate that the coherent resonance peak is a unique feature of the extend $s$-wave pairing as suggested in Refs. 24, 25, and 36. We show that the different pairing symmetries in iron-based superconductors are distinguishable by the distinct features of their corresponding dynamical spin susceptibilities. (2) We investigate the magnetic susceptibility in the mixed SC and 
spin-density wave (SDW) state and find that the coherent peak is strongly weakened in the mixed state. Many experiments suggest a possible coexistence of SC and magnetic ordering in underdoped materials, ${ }^{37-39}$ and the calculated doping dependence of the resonance peak could be checked in future experiments. (3) We show that by considering the RPA correction to the mean-field BCS state, both the $1 / T_{1}$ and the Knight shift show a good agreement with experimental results even when the intraband contributions are included. The RPA correction of the mean-field BCS state can dramatically enhance the interband contribution to the NMR spin-relaxation time $1 / T_{1}$. (4) We show that the dispersion of the magnetic resonance along the $c$ axis observed in neutron scattering experiments ${ }^{28}$ can also be interpreted as indirect evidence of the $s_{x^{2} y^{2}}$ pairing symmetry. The magnetic exchange coupling along $c$ axis has two effects on the resonance: (1) modifying the SC gap along $c$ axis and (2) causing new RPA corrections. Although the second effect produces the right trend of the dispersion, it is too small to explain the dispersion observed in experiments. Therefore, it is the first effect that causes the dispersion. Moreover, in order to explain the experimentally observed dispersion of the resonance peak toward lower energy from $Q_{z}=0$ to $Q_{z}=\pi$, the superconducting order parameters at electron and hole pockets must have opposite values.

\section{MODEL}

The Fe ions form a square lattice in the FeAs layer of LaFeAsO system with As ions sitting in the center of each square plaquette of the Fe lattice and displaced above and below the Fe plane. The crystallographic unit cell includes two Fe and two As ions. We adopt the two orbitals per site model proposed in Ref. 40, capturing the degeneracy of the $d_{x z}$ and $d_{y z}$ orbitals on the Fe atoms and the general location of the Fermi surfaces. The resulting Fermi surface consists of two hole pockets, centered around the $\Gamma=(0,0)$ and $( \pm \pi, \pm \pi)$, and two electron pockets, centered around $( \pm \pi, 0),(0, \pm \pi)$ in the unfolded Brillouin zone [the hole pocket at $( \pm \pi, \pm \pi)$ is an artifact of the two-band model; all the local-density approximation calculations as well as more complicated band models ${ }^{15,41}$ have this pocket at the $\Gamma$ point]. The single electron Hamiltonian is written as

$$
H_{0}=\sum_{\mathbf{k} \sigma} \psi_{\mathbf{k} \sigma}^{\dagger}\left(\begin{array}{cc}
\epsilon_{x}(\mathbf{k})-\mu & \boldsymbol{\epsilon}_{x y}(\mathbf{k}) \\
\epsilon_{x y}(\mathbf{k}) & \boldsymbol{\epsilon}_{y}(\mathbf{k})-\mu
\end{array}\right) \psi_{\mathbf{k} \sigma},
$$

where $\psi_{\mathbf{k} \sigma}^{\dagger}=\left(c_{1, \mathbf{k}, \sigma}^{\dagger}, c_{2, \mathbf{k}, \sigma}^{\dagger}\right)$ is the creation operator with spin $\sigma$ in the orbitals $(1,2)=\left(d_{x z}, d_{y z}\right)$ and $\mu$ is the chemical potential. The electronic dispersions are given by

$$
\begin{gathered}
\epsilon_{x}(\mathbf{k})=-2 t_{1} \cos k_{x}-2 t_{2} \cos k_{y}-4 t_{3} \cos k_{x} \cos k_{y}, \\
\epsilon_{y}(\mathbf{k})=-2 t_{1} \cos k_{y}-2 t_{2} \cos k_{x}-4 t_{3} \cos k_{x} \cos k_{y}, \\
\epsilon_{x y}(\mathbf{k})=-4 t_{4} \sin k_{x} \sin k_{y} .
\end{gathered}
$$

Choosing $t_{1}=-1, t_{2}=1.3$, and $t_{3}=t_{4}=-0.85$ in $\mathrm{eV}$, the eigenvalues of Eq. (1),

$$
E_{ \pm}(\mathbf{k})=\frac{\epsilon_{x}+\epsilon_{y}}{2}-\mu \pm \sqrt{\left(\frac{\epsilon_{x}-\epsilon_{y}}{2}\right)^{2}+\epsilon_{x y}^{2}},
$$

yield the two Fermi pockets aforementioned. For the halffiled system, corresponding to two electrons per site, $\mu$ $=1.54$.

In the following we shall consider the singlet pairing between electrons within each orbital based on $t-J_{1}-J_{2}$ model proposed in Ref. 7. The symmetry of the superconducting order parameter $\Delta(\mathbf{k})$ has two possible $d$-wave types, $d_{x^{2}-y^{2}}$ $=\Delta_{0}\left(\cos k_{x}-\cos k_{y}\right) / 2$ and $d_{x y}=\Delta_{0} \sin k_{x} \sin k_{y}$, and two $s$-wave types, $s_{x^{2}+y^{2}}=\Delta_{0}\left(\cos k_{x}+\cos k_{y}\right) / 2$ and $s_{x^{2} y^{2}}$ $=\Delta_{0} \cos k_{x} \cos k_{y}$. We neglect $d_{x y}$ and the interorbital pairings: Seo et ll. $^{7}$ showed they are negligible for the case of $t-J_{1}-J_{2}$ model. The effective mean-field Hamiltonian is then given by

$$
\begin{gathered}
H_{\mathrm{MF}}=\sum_{\mathbf{k}} \Psi_{\mathbf{k}}^{\dagger} h(\mathbf{k}) \Psi_{\mathbf{k}}, \\
h(\mathbf{k})=\left(\begin{array}{cccc}
\xi_{x}(\mathbf{k}) & \Delta_{1}(\mathbf{k}) & \epsilon_{x y}(\mathbf{k}) & 0 \\
\Delta_{1}^{*}(\mathbf{k}) & -\xi_{x}(\mathbf{k}) & 0 & -\epsilon_{x y}(\mathbf{k}) \\
\epsilon_{x y}(\mathbf{k}) & 0 & \xi_{y}(\mathbf{k}) & \Delta_{2}(\mathbf{k}) \\
0 & -\epsilon_{x y}(\mathbf{k}) & \Delta_{2}^{*}(\mathbf{k}) & -\xi_{x}(\mathbf{k})
\end{array}\right),
\end{gathered}
$$

where $\xi_{x}=\epsilon_{x}-\mu$ and $\xi_{y}=\epsilon_{y}-\mu$ and the four-component spinor $\Psi_{\mathbf{k}}^{\dagger}=\left(c_{1, \mathbf{k}, \uparrow}^{\dagger}, c_{1,-\mathbf{k}, \downarrow}, c_{2, \mathbf{k}, \uparrow}^{\dagger}, c_{2,-\mathbf{k}, \downarrow}\right)$. Due to the $C_{4}$ symmetry of the underlying lattice, $\Delta_{1}(\mathbf{k})=\Delta_{2}(\mathbf{k})$, except for the $d_{x^{2}-y^{2}}$ case, where $\Delta_{1}(\mathbf{k})=-\Delta_{2}(\mathbf{k})$. The $d_{x^{2}-y^{2}}$ and $s_{x^{2}+y^{2}}$ pairing symmetries are nodal and $s_{x^{2} y^{2}}$ is nodeless for any small doping parameter. Note that $s_{x^{2} y^{2}}$ exhibits a sign change between the hole and the electron pockets, while $s_{x^{2}+y^{2}}$ does not.

First we consider the one-loop contribution to the spin susceptibility that includes the intraband and interband contributions as

$$
\chi_{i j}^{\alpha \beta}(q, i \omega)=\int_{0}^{\beta} d \tau\left\langle T S_{i}^{\alpha}(q, \tau) S_{j}^{\beta}(-q, 0)\right\rangle e^{i \omega \tau}
$$

where $S_{i}^{\alpha}(\mathbf{q}, \tau)=\Sigma_{k} c_{\alpha, \mathbf{k}+q, \mu}^{\dagger}(\tau) \sigma_{\mu \nu}^{i} c_{\alpha, \mathbf{k}, \nu}(\tau)$ and $\alpha, \beta$ refer to different orbital indices. The total susceptibility is given by $\chi_{0}^{i j}(\mathbf{q}, i \omega)=\Sigma_{\alpha \beta} \chi_{i j}^{\alpha \beta}(\mathbf{q}, i \omega)$. Since $\chi^{+-}=\left(\chi^{x x}+\chi^{y y}\right) / 2=\chi^{z z}$ for the singlet pairing, we calculate the susceptibility,

$$
\chi_{0}^{z z}(\mathbf{q}, i \omega)=-\frac{T}{2 N} \sum_{\mathbf{k}, n} \operatorname{Tr}\left[G\left(\mathbf{k}+\mathbf{q}, i \omega_{n}+i \omega\right) G\left(\mathbf{k}, i \omega_{n}\right)\right]
$$

where $G(\mathbf{k}, i \omega)=\left(i \omega 1-H_{\mathrm{MF}}\right)^{-1}$. Introducing the band operators, $\Phi_{\mathbf{k}}$, such that $\Psi_{\mathbf{k}}=U(\mathbf{k}) \Phi_{\mathbf{k}}$, the Hamiltonian, $H_{\mathrm{MF}}$, is diagonalized with the eigenvalues, $\mathcal{E}_{i}(\mathbf{k})$, and the eigenvectors, $M_{\alpha \beta}^{i j}(\mathbf{k}, \mathbf{q})$, and the spin susceptibility becomes 


$$
\chi_{0}^{z z}(\mathbf{q}, i \omega)=-\frac{1}{2} \sum_{\mathbf{k}, \alpha, \beta} M_{\alpha \beta}^{i j}(\mathbf{k}, \mathbf{q}) \times\left\{\frac{f\left[\mathcal{E}_{j}(\mathbf{k})\right]-f\left[\mathcal{E}_{i}(\mathbf{k}+\mathbf{q})\right]}{i \omega-\mathcal{E}_{j}(\mathbf{k})+\mathcal{E}_{i}(\mathbf{k}+\mathbf{q})}\right\},
$$

where $f\left[\mathcal{E}_{i}(\mathbf{k})\right]=1 /\left(1+\exp \left[\mathcal{E}_{i}(\mathbf{k}) / T\right]\right)$. For $s$-wave symmetries, $\Delta_{1}=\Delta_{2}$, after the analytical continuation we obtain

$$
\begin{aligned}
\chi_{0}^{z z}(\mathbf{q}, \omega)= & -\frac{1}{2} \sum_{\mathbf{k}, \mu \nu= \pm}\left[1-\frac{\Delta(\mathbf{k}) \Delta(\mathbf{k}+\mathbf{q})+E_{\mu}(\mathbf{k}) E_{\nu}(\mathbf{k}+\mathbf{q})}{\mathcal{E}_{\mu}(\mathbf{k}) \mathcal{E}_{\nu}(\mathbf{k}+\mathbf{q})}\right] \\
& \times \frac{f\left[\mathcal{E}_{\mu}(\mathbf{k})\right]-f\left[-\mathcal{E}_{\nu}(\mathbf{k}+\mathbf{q})\right]}{\omega+i \eta-\mathcal{E}_{\mu}(\mathbf{k})-\mathcal{E}_{\nu}(\mathbf{k}+\mathbf{q})},
\end{aligned}
$$

where $\mathcal{E}_{ \pm}(\mathbf{k})=\sqrt{E_{ \pm}^{2}(\mathbf{k})+\Delta^{2}(\mathbf{k})}$.

Within RPA, the spin susceptibility $\chi_{\mathrm{RPA}}(\mathbf{q}, \omega)$ is given in matrix form by

$$
\chi_{\mathrm{RPA}}(\mathbf{q}, i \omega)=\left[\mathbf{I}-\Gamma \chi_{0}(\mathbf{q}, i \omega)\right]^{-1} \chi_{0}(\mathbf{q}, i \omega),
$$

where $\mathbf{I}$ is a unit matrix and the vertex $\Gamma=U \mathbf{I}$ with the value of $U$ chosen in the paramagnetic phase. $\chi_{0}$ above is written in $2 \times 2$ matrix form whose entries contain the orbital contributions of Eq. (7). Note that the RPA enhancement of the spin fluctuations is determined by the $\operatorname{det}\left|\mathbf{I}-\Gamma \chi_{0}(\mathbf{q}, i \omega)\right|$. In the superconducting state the quasiparticles at the hole and electron Fermi surfaces are connected by the $\mathbf{Q}=(\pi, 0)$, $(0, \pi)$ wave vector. In the unfolded BZ, the $s_{x^{2} y^{2}}$ order parameter satisfies the condition $\Delta(\mathbf{k})=-\Delta(\mathbf{k}+\mathbf{Q})$. The imaginary part of the interband bare susceptibility is zero for small frequencies due to the opening of the gap and experiences a discontinuous jump at $\omega_{c} \sim\langle|\Delta(\mathbf{k})|+|\Delta(\mathbf{k}+\mathbf{Q})|\rangle$.

\section{COMPARISON OF SPIN SUSCEPTIBILITY IN DIFFERENT PAIRING SYMMETRY STATES}

Figure 1 presents the imaginary part of the RPA spin susceptibility $\operatorname{Im} \chi_{\mathrm{RPA}}(\mathbf{Q}, \omega)$ for various superconducting order parameters on (a) the electron-doped side and (b) the holedoped side. We observe that only the $s_{x^{2} y^{2}}$ pairing symmetry gives peaks unsuppressed by RPA. At both electron and hole dopings, there are two pronounced peaks for this pairing symmetry. While the number of peaks is model dependent and in this case characteristic to the two-band model used, the high intensity of the peaks is a generic feature stemming from the coherence factor $\left[1-\Delta(\mathbf{k}) \Delta(\mathbf{k}+\mathbf{Q}) / \mathcal{E}_{+}(\mathbf{k}) \mathcal{E}_{-}(\mathbf{k}\right.$ $+\mathbf{Q})] / 2$, where $\mathbf{Q}=(\pi, 0)$. This coherence factor clearly becomes large if $\Delta(\mathbf{k})=-\Delta(\mathbf{k}+\mathbf{Q})$, a condition only met by the $s_{x^{2} y^{2}}$ pairing. The coherence factor is weighted by the spectra weight $\delta\left[\omega-\mathcal{E}_{+}(\mathbf{k})-\mathcal{E}_{-}(\mathbf{k}+\mathbf{Q})\right]$ and integrated over $\mathbf{k}$ to obtain the final $\operatorname{Im}(\chi)$. For the $s_{x^{2} y^{2}}$ symmetry pairing, the energy profile (after the SC gap opening) is almost isotropic and flat around the $\Gamma$ and $X$ points. This means that at $\omega$ $\sim \omega_{c}$, almost all the $k$ 's around the electron and hole FSs can contribute to the coherent peak, making its intensity significantly higher than the other pairing symmetries. For $d_{x^{2}-y^{2}}$ $\left(s_{x^{2}+y^{2}}\right)$ pairing symmetries, there are four nodes on around $\Gamma$ $(X)$ point, making the energy profile around the $\Gamma(X)$ point considerably angle dependent. Therefore it is rather difficult to find a constant $\omega_{c}$ such that most k's on the FSs can

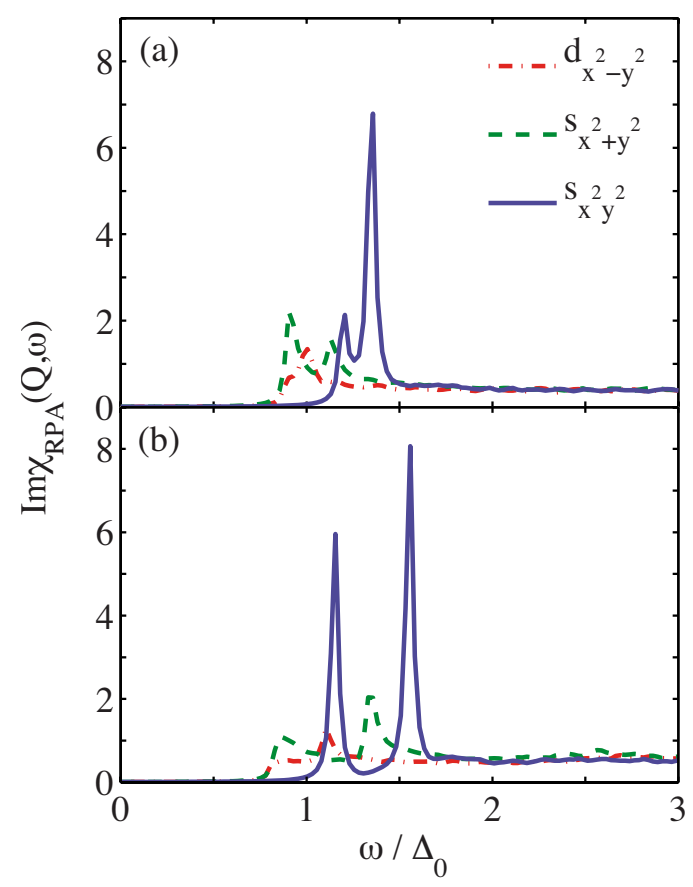

FIG. 1. (Color online) The imaginary part of the RPA spin susceptibility $\chi_{\mathrm{RPA}}$ at $\mathbf{Q}=(\pi, 0)$ for various superconducting pairing symmetries. (a) Hole-doped side $(\mu=1.4)$ and (b) electron-doped side $(\mu=1.6)$. The blue line represents the result for $s_{x^{2} y^{2}}$ $\sim \cos k_{x} \cos k_{y}$, the green dashed line represents the $s_{x^{2}+y^{2}} \sim \cos k_{x}$ $+\cos k_{y}$, and the red dashed line represents the $d_{x^{2}-y^{2}}$ symmetry. We used $\Delta_{0}=0.3$ and $U=0.6 t_{1}$.

contribute to the sum. Apart from the number of peaks, the previous discussion is generic and does not depend on the particular model used. Within the two-band model, the number of peaks for the $s_{x^{2} y^{2}}$ pairing symmetry is 2 . This is due to the FS topology given by the two-band model. As shown in Fig. 2 the two resonance peaks correspond to the nesting of two pairs of electron-hole pockets: the nesting between $\Gamma$ hole pocket and $X$ electron pocket and the nesting between $M$ hole pocket and $Y$ electron pocket. When doping is small, we can roughly express the two resonance frequencies as

$$
\omega_{\text {res }, 1} \sim\left|\Delta\left(\mathbf{k}_{1}^{h}\right)\right|+\left|\Delta\left(\mathbf{k}_{1}^{e}\right)\right|,
$$

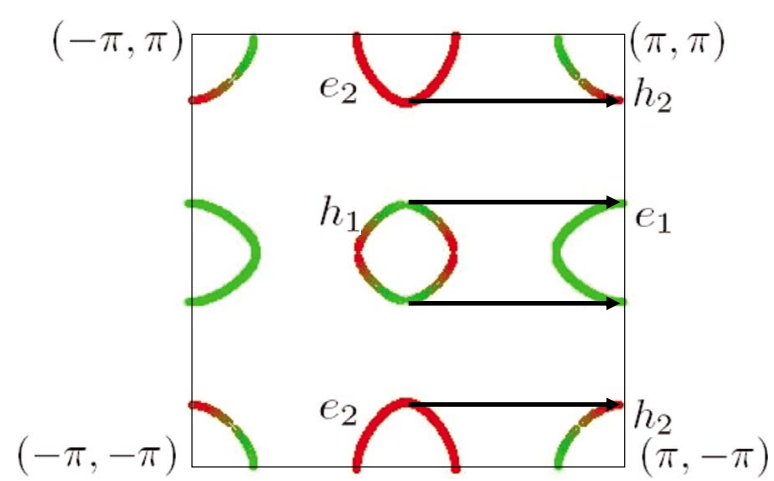

FIG. 2. (Color online) The schematic of the FSs not far away from half-filling. The hole and electron pockets are connected by the vector $\mathbf{Q}$. The color indicates the orbits, green $=y z$ and red $=x z$. 


$$
\omega_{\text {res, } 2} \sim\left|\Delta\left(\mathbf{k}_{2}^{h}\right)\right|+\left|\Delta\left(\mathbf{k}_{2}^{e}\right)\right| .
$$

According to the pairing gap formula $\cos \left(k_{x}\right) \cos \left(k_{y}\right)$, the larger FS gives a smaller gap magnitude. Therefore we have $\omega_{\text {res, } 1}>\omega_{\text {res,2 }}$ because the FS around $\Gamma$ is larger than that around $M$. For the $d_{x^{2}-y^{2}}$ pairing, the two hole pockets have nodes and the minimum gaps $\Delta\left(\mathbf{k}_{i}^{h}\right)$ 's on the hole surface are hence much smaller than the electron surface gaps $\Delta\left(\mathbf{k}_{i}^{e}\right)^{\prime}$ 's; for the $s_{x^{2}+y^{2}}$ pairing, the two electron pockets have nodes and the gap values on the electron pockets are much smaller. Hence, the peaks for these two pairing symmetries shift to the lower energy than to those of the $s_{x^{2} y^{2}}$ symmetry.

The band structure of the current two-band model as the function of doping will not exactly represent the real materials. However, the differences of the resonance peaks between electron doped and hole doped strongly suggest that the resonance peak is tied to the combination of the opposite signs of SC order parameters and the close matching of Fermi surfaces between electron and hole pockets. The resonance is stronger for better nested surfaces connected by $Q$ vectors. This provides a direct explanation of the orbital selective electron-mode coupling observed in angle resolved photoemission experiments. ${ }^{42}$

\section{MAGNETIC RESONANCE IN THE MIXED SC AND SDW STATES WITH $s_{x^{2} y^{2}}$ PAIRING SYMMETRY}

Recently, several experiments indicate the coexistence of SC and SDW orderings in underdoped materials. ${ }^{37-39}$ Therefore, it is of great interest to investigate whether the magnetic resonance survives in the mixed state. Treating the SDW order in mean field, we now have off-diagonal terms between $\mathbf{k}$ and $\mathbf{k}+\mathbf{Q}_{\mathrm{SDW}}$, where $\mathbf{Q}_{\mathrm{SDW}}=(\pi, 0)$. In the mean-field approximation, the spin exchange interaction,

$$
H_{\mathrm{ex}}=\sum_{\mathbf{q}=\left(q_{x}, q_{y}\right)} J(\mathbf{q}) \mathbf{S}(\mathbf{q}) \cdot \mathbf{S}(-\mathbf{q}),
$$

can be decoupled as

$$
H_{\mathrm{ex}}^{\mathrm{MF}}=\sum_{\mathbf{q}} J(\mathbf{q})\langle\mathbf{S}(\mathbf{q})\rangle \cdot \mathbf{S}(-\mathbf{q})+\text { H.c. },
$$

where $J(\mathbf{q})=J_{1}\left[\cos \left(q_{x}\right)+\cos \left(q_{y}\right)\right]+2 J_{2} \cos \left(q_{x}\right) \cos \left(q_{y}\right)$. Substituting the realistic magnetic structure, $\langle\mathbf{S}(\mathbf{q})\rangle$ $=(0,0, M) \delta_{\mathbf{q}, \mathbf{Q}_{\mathrm{SDW}}}$, the mean-field interaction reduces to

$$
H_{\mathrm{ex}}^{\mathrm{MF}}=W \sum_{i \neq j} c_{i, \mathbf{k}, \sigma}^{\dagger} \tau_{z}^{\sigma \sigma^{\prime}} c_{j, \mathbf{k}+\mathbf{Q}_{\mathrm{SDW}}, \sigma^{\prime}}+\text { H.c. },
$$

where $W=J\left(\mathbf{Q}_{\mathrm{SDW}}\right) M$ is a control parameter chosen between $0 \sim \Delta_{0}$ in our calculation. Therefore we can write the full mean-field Hamiltonian as

$$
H^{\mathrm{MF}}=\sum_{\mathbf{k}} \boldsymbol{\Psi}_{\mathbf{k}}^{\dagger} \tilde{h}(\mathbf{k}) \boldsymbol{\Psi}_{\mathbf{k}}
$$

where

$$
\begin{aligned}
\tilde{h}(\mathbf{k})= & h(\mathbf{k}) \otimes\left(I+\tau_{z}\right) / 2+h\left(\mathbf{k}+\mathbf{Q}_{\mathrm{SDW}}\right) \otimes\left(I-\tau_{z}\right) / 2 \\
& +W I \otimes I \otimes \tau_{x},
\end{aligned}
$$

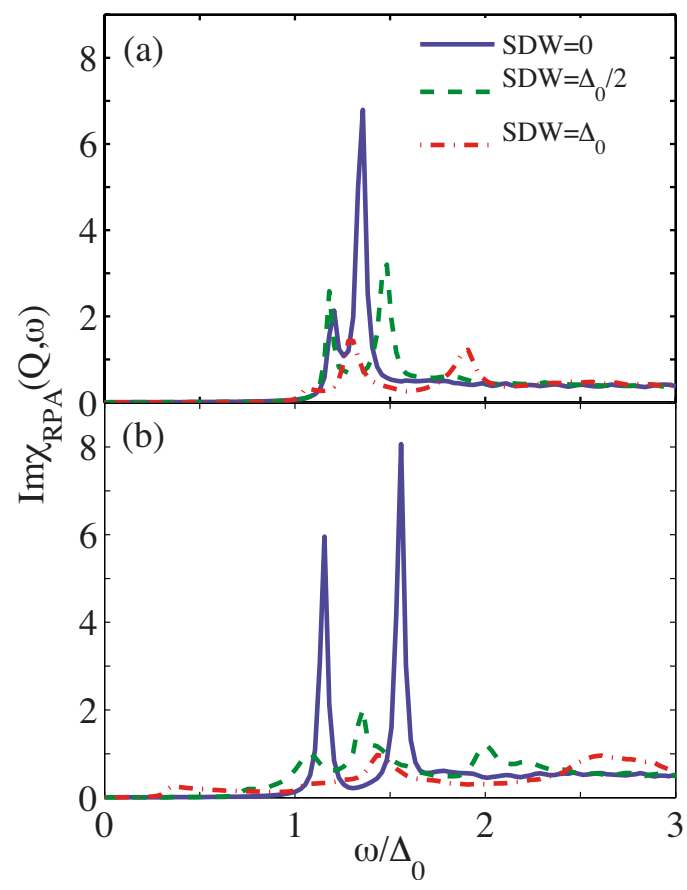

FIG. 3. (Color online) The imaginary part of the RPA susceptibility in the (a) hole-doped and (b) electron-doped sides. We used the same $\Delta_{0}$ and the chemical potentials with Fig. 1 .

$$
\begin{aligned}
\boldsymbol{\Psi}_{\mathbf{k}}^{\dagger}= & \left\{c_{1, \mathbf{k}, \uparrow}^{\dagger}, c_{1, \mathbf{k}+\mathbf{Q}_{\mathbf{S D W}}^{\dagger}, \uparrow}^{\dagger}, c_{1,-\mathbf{k}, \downarrow},\right. \\
& \left.c_{1,-\mathbf{k}+\mathbf{Q}_{\mathbf{S D W}}, \downarrow}, c_{2, \mathbf{k}, \uparrow}^{\dagger}, c_{2, \mathbf{k}+\mathbf{Q}_{\mathbf{S D W}}, \uparrow}^{\dagger}, c_{2,-\mathbf{k}, \downarrow}, c_{2,-\mathbf{k}+\mathbf{Q}_{\mathbf{S D W}}, \downarrow}\right\},
\end{aligned}
$$

with $I$ as a $2 \times 2$ identity matrix and $h(\mathbf{k})$ as a $4 \times 4$ matrix given in Eq. (5).

Figure 3 presents the RPA total spin susceptibility in the mixed state of SDW coexisting with $s_{x^{2} y^{2}}$ order in the (a) hole-doped and (b) electron-doped sides. The pronounced resonance peaks observed in the pure $s_{x^{2} y^{2}}$ superconducting state are reduced by the presence of the SDW. The SDW order doubles the unit cell and reduces the original Brillouin zone which causes the weight of the spin susceptibility at $(0, \pi)$ to spread out.

\section{NMR SPIN-RELAXATION RATE AND KNIGHT SHIFT}

As discussed earlier in the paper, the experimental absence of a coherent peak and the presence of near cubic power-law dependence on temperature of the NMR spinrelaxation rate $1 / T_{1}$ in these superconductors ${ }^{29-32}$ have been a challenge for theories predicting the extended $s$-wave pairing symmetry. In a previous paper, ${ }^{33}$ two of us also calculated the NMR spin-relaxation rate $1 / T_{1}$ of the bare superconductor and found that it factorizes into interband and intraband contributions. While, for the $\cos \left(k_{x}\right) \cos \left(k_{y}\right)$ order parameter, the interband contribution to the NMR spinrelaxation rate does not exhibit a coherence peak, the intraband contribution is larger than the interband contribution and still exhibits an enhancement right below $T_{c}$ owing to its fully gapped $s$-wave nature. Adding the two contributions we 


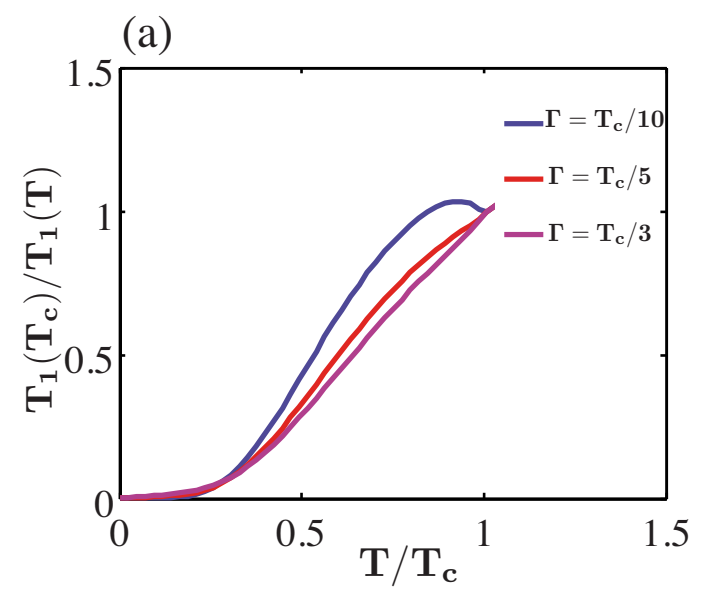

(b)

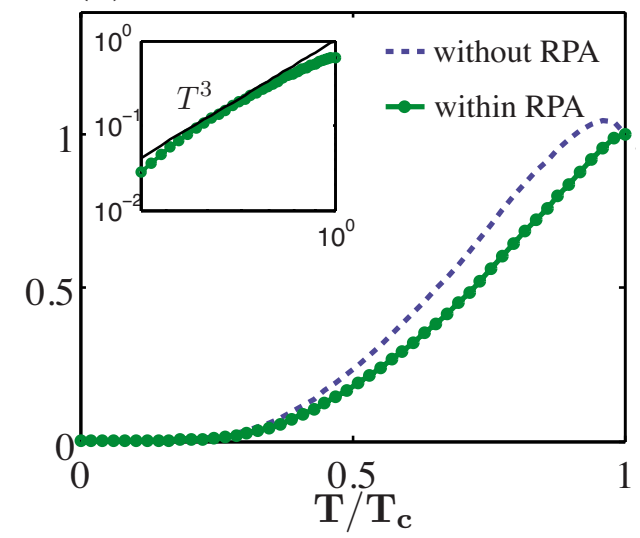

FIG. 4. (Color online) (a) The NMR spin-relaxation time $1 / T_{1}$ as a function of temperature $T$ in unit of $T_{c}$ for various values of $\Gamma$, which is considered as the phenomenological disorder parameter. We used $\Delta_{0} / 2=T_{c}$ with $\Delta_{0}=0.3$. (b) The effect of the spin fluctuation within RPA susceptibility. We choose the on-site Coulomb repulsion $U=0.5 t_{1}$ such that the system remains in the paramagnetic state. Inset: the log-log plot of the RPA susceptibility with line of $T^{3}$ showing the temperature dependence of $\chi_{\mathrm{RPA}}$ just below $T_{c}$.

find that, although the coherence peak for $\cos \left(k_{x}\right) \cos \left(k_{y}\right)$ is smaller than that for a sign-preserving gap [such as, for example, $\left.\left|\cos \left(k_{x}\right) \cos \left(k_{y}\right)\right|\right]$, it is still present due to the intraband contribution. The coherence peak can be strongly reduced if the intraband scattering is stronger than interband scattering. Here we also show that the NMR experimental results are consistent with the extended $s$-wave pairing symmetry if the simple RPA correction over the mean-field bare SC state is considered as argued in Ref. 34.

The spin-relaxation rate at a temperature $T$ is defined as

$$
R=\frac{1}{T_{1} T}=-\frac{1}{2 \pi} \lim _{\omega_{0} \rightarrow 0} \frac{\operatorname{Im} \sum_{\mathbf{q}} A(\mathbf{q}) \chi^{+-}\left(\mathbf{q}, \omega_{0}\right)}{\omega_{0}}
$$

where $A(\mathbf{q})$ is a structure factor: for $\mathrm{F}$ it is roughly isotropic, while for As, $A(\mathbf{q})=\cos q_{x} / 2 \cos q_{y} / 2$ (neglecting the fact that the position of As is out of plane of Fe's). In the following we take the structure factor uniform, i.e., $A(\mathbf{q})=1$.

In Fig. 4, we present the NMR spin-relaxation time as a

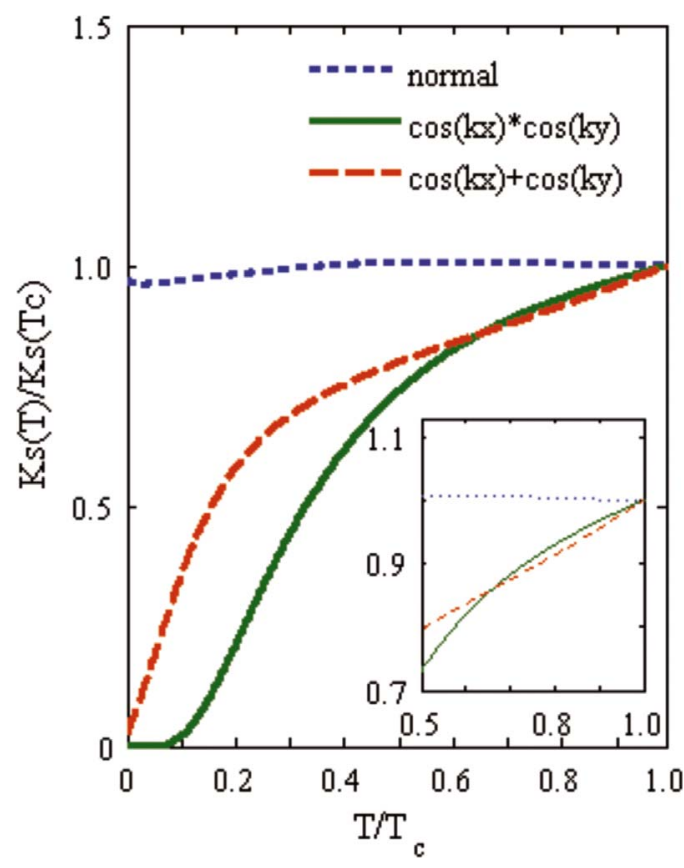

FIG. 5. (Color online) Temperature dependence of the Knight shift. The solid green line is a plot with a sign-reversed extended $s$ wave, $\cos k_{x} \cos k_{y}$, and the dashed red line is one with signpreserved extended $s$ wave, $\cos k_{x}+\cos k_{y}$. Inset shows the difference between two order parameters just below $T_{c}$.

function of the temperature (in unit of $T_{c}$ ). The coherence peak is present in the bare $s_{x^{2} y^{2}}$ pairing SC state if the impurity effect is weak, as found in Ref. 33. The coherence peak is reduced by the effects of disorder and the RPA correction. The disorder is taken in account by broadening the imaginary part of frequency, $\Gamma$. Figure 4(a) shows that the coherence peak at $T_{c}$ is reduced as disorder increases, $\Gamma=T_{c} / 10$ $\sim T_{c} / 3$. Figure 4(b) presents the effect of the RPA correction. A small interaction $U \sim 0.5 t_{1}$ is enough to suppress the coherence peak.

We also calculate the Knight shift, $K_{s}=\chi(\mathbf{q} \rightarrow 0, \omega=0)$. Figure 5 plots the Knight shift value as a function of temperature in the different $s$-wave pairing symmetry states: a sign-reversed extended $s$ wave $\left(s_{x^{2} y^{2}} \sim \cos k_{x} \cos k_{y}\right)$ and an extended $s$ wave $\left(s_{x^{2}+y^{2}} \sim \cos k_{x}+\cos k_{y}\right)$. The main difference between these two ordering symmetry states is that the former one is fully gapped while the second one has nodes on the electron pockets. At low temperatures, $K_{s}(T)$ has a power-law temperature dependence for the second case. The fact that $K_{s}(T)$ is a concave function around $T=0.4 T_{c}$ indicates the presence of two-gap values, as observed in experiments. $^{29}$

\section{EFFECT OF MAGNETIC EXCHANGE COUPLING ALONG $c$ AXIS}

Now we turn to the effects of the spin fluctuations between the FeAs layers. For the 122 series of iron-based superconductors, the coupling between FeAs layers along $c$ axis is very important. In the parent compounds, the magnetic exchange coupling $S J_{z}$ along $c$ axis determined by neu- 
tron scattering experiments is around $6 \mathrm{meV}$-around one fifth of the in-plane magnetic exchange coupling; ${ }^{43}$ this suggests a strong three-dimensional (3D) electronic structure. The measurement of spin excitation resonance peaks in $\mathrm{BaFe}_{1.9} \mathrm{Ni}_{0.1} \mathrm{As}_{2}$ shows that they are dispersive along $c$-axis direction, and the resonance peak at $3 \mathrm{D}$ antiferromagnetic (AF) ordering wave vector $(\pi, 0, \pi)$ is $\hbar \omega=7 \mathrm{meV}$ below $T_{c}$ different from one at $\mathbf{Q}_{1}=(\pi, 0,0), \hbar \omega=9.1 \mathrm{meV} .{ }^{28}$

The Hamiltonian for the antiferromagnetic exchange coupling in $c$ axis can be generally written as

$$
H_{\mathrm{int}}=J_{z} \sum_{\mathbf{r}, \alpha} \mathbf{S}^{\alpha}(\mathbf{r}) \cdot \mathbf{S}^{\alpha}(\mathbf{r} \pm \hat{z})+J_{z}^{\prime} \sum_{\mathbf{r}, \alpha \neq \beta} \mathbf{S}^{\alpha}(\mathbf{r}) \cdot \mathbf{S}^{\beta}(\mathbf{r} \pm \hat{z}),
$$

where $J_{z}$ is a spin coupling constant for the intraorbital and $J_{z}^{\prime}$ is a spin coupling constant for interorbital between the adjacent FeAs layers.

In general, there are two possible effects generated by this spin coupling. First, it could produce the variation in the superconducting gap as a function of momentum wave vector along $c$ axis. This effect has been used to explain the dispersion of magnetic resonance. ${ }^{28}$ Here we point out that the explanation is only consistent with the extended $s_{x^{2} y^{2}}$ wave. Let us revisit the analysis given in Ref. 28 . Let $\Delta_{0}^{e}$ and $\Delta_{0}^{h}$ denote the superconducting gaps on the hole and electron pockets, respectively (in a pure two-dimensional model). By considering the antiferromagnetic coupling between layers, the gap functions, at the mean-field level, are naturally modified to $\Delta_{e}\left(k_{z}\right)=\Delta_{e}^{0}+\delta \cos \left(k_{z}\right)$ and $\Delta_{h}\left(k_{z}\right)=\Delta_{h}^{0}+\delta \cos \left(k_{z}\right)$. For a $S^{ \pm}$pairing symmetry, $\Delta_{0}^{e} \sim-\Delta_{0}^{h} \sim-\Delta_{0}$. Therefore the dispersion of the resonance along $c$ axis is roughly determined by

$$
\begin{aligned}
\hbar \omega\left(q_{z}\right) \sim & \min \left[\left\langle\left|\Delta_{e}\left(k_{z}\right)\right|+\left|\Delta_{h}\left(k_{z}+q_{z}\right)\right|\right\rangle, k_{z}\right] \sim 2 \Delta_{0} \\
& -2 \delta\left|\sin \left(\frac{q_{z}}{2}\right)\right| .
\end{aligned}
$$

In the above analysis, if we assume the sign of gap does not change from electron to hole pockets, it is easy to see that the dispersion of the resonance in Eq. (20) changes to $\hbar \omega\left(q_{z}\right)$ $\sim 2 \Delta_{0}+2 \delta\left|\sin \left(\frac{q_{z}}{2}\right)\right|$, which will result in an opposite dispersion of the resonance energy than that reported in the experiments: larger at the wave vector $(\pi, 0, \pi)$ than at the wave vector $(\pi, 0,0)$, obviously contradicting the experimental results.

There is also a second effect due to the magnetic exchange coupling along $c$ axis, namely, a simple RPA correction to spin susceptibility due to the presence of the exchange coupling. It is easy to show that in the presence of above exchange coupling, the RPA spin susceptibility is modified to

$$
\chi^{\mathrm{RPA}}(\mathbf{q}, \omega)=\sum_{\alpha \beta}\left[\chi_{0}(\mathbf{q}, \omega)\left[1-V(\mathbf{q}) \chi_{0}(\mathbf{q}, \omega)\right]^{-1}\right]_{\alpha \beta},
$$

with the vertex
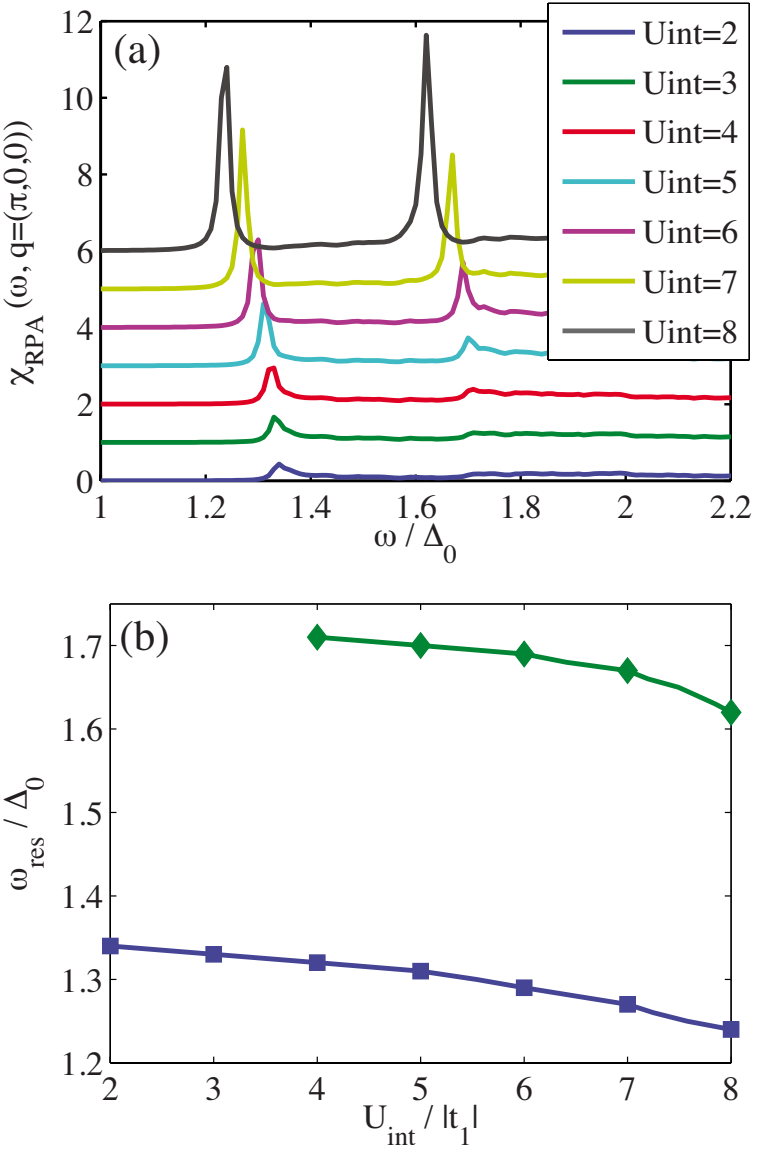

FIG. 6. (Color online) (a) The RPA spin susceptibilities in the electron-doped $s_{x^{2} y^{2}}$ superconducting state $\left(\mu=1.65\left|t_{1}\right|\right)$ with a various $U_{\text {int }}(\mathbf{q})$ (in increasing order from the lowest to highest curves) and (b) the dispersion of $\omega_{\text {res }}$ as a function of $U_{\text {int }}(\mathbf{q})$.

$$
V(\mathbf{q})=\left(\begin{array}{cc}
U_{\mathrm{int}}(\mathbf{q}) & -J_{z}^{\prime} \cos q_{z} \\
-J_{z}^{\prime} \cos q_{z} & U_{\mathrm{int}}(\mathbf{q})
\end{array}\right),
$$

where $U_{\text {int }}(\mathbf{q})=U-J_{z} \cos q_{z}$ with $U$ as a onsite Coulomb repulsion. We calculate $\chi_{\mathrm{RPA}}(\omega, \mathbf{q})$ in the electron-doped superconducting state $\left(\mu=1.65\left|t_{1}\right|\right)$ with $s_{x^{2} y^{2}}$ pairing symmetry, $\Delta(\mathbf{k})=\Delta_{0} \cos k_{x} \cos k_{y} . J_{z}^{\prime}$, the interorbital coupling, has very little effect on the positions of $\omega_{\text {res }}$. Therefore, we only need to consider the intraorbital spin coupling between the layers, $J_{z}$. The effect of $J_{z}$ simply creates an effective $U_{\text {int }}(q)$ which is larger at $q=(\pi, 0, \pi)$ than at $q=(\pi, 0, \pi)$. Figure 6(b) displays the dispersion of $\omega_{\text {res }}$ as a function of $U_{\text {int }}(\mathbf{q})$. It is clear that the resonance energy decreases as $U_{\text {int }}$ increases. Therefore, in general, the RPA correction would result in a dispersion of resonance peak that has a correct trend as the experimental results. However, it is also clear from Fig. 6 that $J_{z}$ has to be comparable with $U$ in order to create a visible dispersion. Since $J_{z}$ is really a small energy scale compared to both bandwidth and interaction, the dispersion due to RPA correction from $J_{z}$ is negligible.

From above analysis, we can conclude that the dispersion of the resonance peak along $c$ axis mainly stems from the modification the SC gap along $c$ axis. The fact that the dispersion of the resonance peak shifts toward lower energy 
from $Q_{z}=0$ to $Q_{z}=\pi$ indicates that the superconducting order parameters at electron and hole pockets must have opposite values. There are also two predictions drawn from this analysis. (1) It predicts $\delta / \Delta_{0} \sim J_{z} / J_{2}$ if the superconducting is driven by magnetic exchange. This quantitative relation has been confirmed in Ref. 28. (2) Since it is believed that the 1111 series of iron pnictides are more two dimensional than the 122 series, this analysis will predict very little dispersion of the magnetic resonance along $c$ axis in the 1111 series.

\section{CONCLUSION}

We have investigated the magnetic properties in the SC states of the iron-based superconductors based on a twoorbital model, identifying generic, model-independent, experimentally observable predictions. We have found the $s_{x^{2} y^{2}}$ pairing symmetry exhibits a strong magnetic resonance, which is absent from other pairing symmetries (the similar result was also reached in Ref. 44 when this paper was prepared). We have predicted that the coexistence of SDW order with the SC order weakens the resonance peak. We have shown a good agreement with NMR experimental results; both the $1 / T_{1}$ and the Knight shift can be reached after considering the RPA correction from a bare BCS state with the extended $s_{x^{2} y^{2}}$ pairing symmetry. We have also explicitly studied the dispersion of magnetic resonance along $c$ axis ${ }^{28}$ and have concluded that the dispersion is mainly caused by the modification of SC gap along $c$ axis, and it indicates that the superconducting order parameters at electron and hole pockets must have opposite values. This explanation is also consistent with the three-dimensional nature of the 122 series of iron pnictides as shown in different experiments. ${ }^{43,45} \mathrm{Fi}$ nally, we also want to point out that this explanation does not contradict the possible existence of gap nodes along $c$ axis which may explain many nodal-like experimental results in the 122 materials ${ }^{46,47}$ since the resonance peak is determined by the summation of the absolute values of SC gaps at electron and hole pockets.

\section{ACKNOWLEDGMENTS}

J.H. thanks Pengcheng Dai, W. Tsai, and D. X. Yao for useful discussions. B.A.B. thanks P. W. Anderson and N. P. Ong for useful discussions. J.H., K.S., and C.F. were supported by the NSF under Grant No. PHY-0603759.
${ }^{1}$ Y. Kamihara, T. Watanabe, M. Hirano, and H. Hosono, J. Am. Chem. Soc. 130, 3296 (2008).

${ }^{2}$ H. Takahashi, K. Igawa, K. Arii, Y. Kamihara, M. Hirano, and H. Hosono, Nature (London) 453, 376 (2008).

${ }^{3}$ G. F. Chen, Z. Li, D. Wu, G. Li, W. Z. Hu, J. Dong, P. Zheng, J. L. Luo, and N. L. Wang, Phys. Rev. Lett. 100, 247002 (2008).

${ }^{4}$ X. H. Chen, T. Wu, G. Wu, R. H. Liu, H. Chen, and D. F. Fang, Nature (London) 453, 761 (2008).

${ }^{5}$ H.-H. Wen, G. Mu, L. Fang, H. Yang, and X. Zhu, EPL 82, 17009 (2008).

${ }^{6}$ H. A. Mook, Y. Sidis, B. Fauqué, V. Balédent, and P. Bourges, Phys. Rev. B 78, 020506(R) (2008).

${ }^{7}$ K. Seo, B. A. Bernevig, and J. Hu, Phys. Rev. Lett. 101, 206404 (2008).

${ }^{8}$ I. I. Mazin, D. J. Singh, M. D. Johannes, and M. H. Du, Phys. Rev. Lett. 101, 057003 (2008).

${ }^{9}$ F. Wang, H. Zhai, Y. Ran, A. Vishwanath, and D.-H. Lee, Phys. Rev. Lett. 102, 047005 (2009).

${ }^{10}$ C. Fang, H. Yao, W.-F. Tsai, J. P. Hu, and S. A. Kivelson, Phys. Rev. B 77, 224509 (2008).

${ }^{11}$ T. Yildirim, Phys. Rev. Lett. 101, 057010 (2008).

${ }^{12}$ Q. Si and E. Abrahams, Phys. Rev. Lett. 101, 076401 (2008).

${ }^{13}$ F. Ma, Z.-Y. Lu, and T. Xiang, Phys. Rev. B 78, 224517 (2008).

${ }^{14}$ C. Xu, M. Mueller, and S. Sachdev, Phys. Rev. B 78, 020501(R) (2008).

${ }^{15}$ V. Cvetkovic and Z. Tesanovic, EPL 85, 37002 (2009).

${ }^{16}$ H. Ding, P. Richard, K. Nakayama, T. Sugawara, T. Arakane, Y. Sekiba, A. Takayama, S. Souma, T. Sato, T. Takahashi, Z. Wang, X. Dai, Z. Fang, G. F. Chen, J. L. Luo, and N. L. Wang, EPL 83, 47001 (2008).

${ }^{17}$ K. Nakayama, T. Sato, P. Richard, Y.-M. Xu, Y. Sekiba, S. Souma, G. F. Chen, J. L. Luo, N. L. Wang, H. Ding, and T.
Takahashi, EPL 85, 67002 (2009).

${ }^{18}$ L. Wray, D. Qian, D. Hsieh, Y. Xia, L. Li, J. Checkelsky, A. Pasupathy, K. Gomes, A. Fedorov, G. Chen, J. Luo, A. Yazdani, N. Ong, N. Wang, and M. Hasan, arXiv:0808.2185 (unpublished).

${ }^{19}$ W.-F. Tsai, D.-X. Yao, B. Andrei Bernevig, and JiangPing Hu, arXiv:0812.0661 (unpublished).

${ }^{20}$ P. Ghaemi, F. Wang, and A. Vishwanath, Phys. Rev. Lett. 102, 157002 (2009)

${ }^{21}$ D. Parker and I. Mazin, arXiv:0812.4416 (unpublished).

${ }^{22}$ J. Wu and P. Phillips, Phys. Rev. B 79, 092502 (2009).

${ }^{23}$ Y.-Y. Zhang, C. Fang, X. Zhou, K. Seo, W.-F. Tsai, B. Andrei Bernevig, and J. Hu, arXiv:0903.1694 (unpublished).

${ }^{24}$ T. A. Maier and D. J. Scalapino, Phys. Rev. B 78, 020514(R) (2008).

${ }^{25}$ M. M. Korshunov and I. Eremin, Phys. Rev. B 78, 140509(R) (2008).

${ }^{26}$ A. Christianson, E. Goremychkin, R. Osborn, S. Rosenkranz, M. Lumsden, C. Malliakas, I. Todorov, H. Claus, D. Chung, M. Kanatzidis, R. Bewley, and T. Guidi, Nature (London) 456, 930 (2008).

${ }^{27}$ M. Lumsden, A. Christianson, D. Parshall, M. Stone, S. E. Nagler, G. MacDougall, H. Mook, K. Lokshin, T. Egami, D. Abernathy, E. Goremychkin, R. Osborn, M. McGuire, A. Sefat, R. Jin, B. Sales, and D. Mandrus, Phys. Rev. Lett. 102, 107005 (2009).

${ }^{28}$ S. Chi, A. Schneidewind, J. Zhao, L. W. Harriger, L. Li, Y. Luo, G. Cao, Z. Xu, M. Loewenhaupt, J. Hu, and P. Dai, Phys. Rev. Lett. 102, 107006 (2009).

${ }^{29}$ K. Matano, Z. A. Ren, X. L. Dong, L. L. Sun, Z. X. Zhao, and G.-q. Zheng, EPL 83, 57001 (2008).

${ }^{30}$ N. Terasaki, H. Mukuda, M. Yashima, Y. Kitaoka, K. Miyazawa, 
P. Shirage, H. Kito, H. Eisaki, and A. Iyo, J. Phys. Soc. Jpn. (to be published).

${ }^{31}$ H. Fukazawa, T. Yamazaki, K. Kondo, Y. Kohori, N. Takeshita, P. Shirage, K. Kihou, K. Miyazawa, H. Kito, H. Eisaki, and A. Iyo, J. Phys. Soc. Jpn. 78, 033704 (2009).

${ }^{32}$ H. Mukuda, N. Terasaki, M. Yashima, H. Nishimura, Y. Kitaoka, and Y. Iyo, arXiv:0901.4607 (unpublished).

${ }^{33}$ M. M. Parish, J. Hu, and B. A. Bernevig, Phys. Rev. B 78, 144514 (2008).

${ }^{34}$ D. Parker, O. V. Dolgov, M. M. Korshunov, A. A. Golubov, and I. I. Mazin, Phys. Rev. B 78, 134524 (2008).

${ }^{35}$ M. S. Laad and L. Craco, arXiv:0902.3400 (unpublished).

${ }^{36}$ M. Daghofer, A. Moreo, J. A. Riera, E. Arrigoni, D. J. Scalapino, and E. Dagotto, Phys. Rev. Lett. 101, 237004 (2008).

${ }^{37}$ R. Khasanov, H. Luetkens, A. Amato, H.-H. Klauss, Z.-A. Ren, J. Yang, W. Lu, and Z.-X. Zhao, Phys. Rev. B 78, 092506 (2008).

${ }^{38}$ A. J. Drew, F. L. Pratt, T. Lancaster, S. J. Blundell, P. J. Baker, R. H. Liu, G. Wu, X. H. Chen, I. Watanabe, V. K. Malik, A. Dubroka, K. W. Kim, M. Rossle, and C. Bernhard, Phys. Rev. Lett. 101, 097010 (2008).

${ }^{39}$ Y. Xiao, Y. Su, R. Mittal, T. Chatterji, T. Hansen, C. M. N.
Kumar, S. Matsuishi, H. Hosono, and T. Brueckel, Phys. Rev. B 79, 060504(R) (2009).

${ }^{40}$ S. Raghu, X.-L. Qi, C.-X. Liu, D. Scalapino, and S.-C. Zhang, Phys. Rev. B 77, 220503(R) (2008).

${ }^{41}$ K. Kuroki, S. Onari, R. Arita, H. Usui, Y. Tanaka, H. Kontani, and H. Aoki, Phys. Rev. Lett. 101, 087004 (2008).

${ }^{42}$ P. Richard, T. Sato, K. Nakayama, S. Souma, T. Takahashi, Y.-M. Xu, G. F. Chen, J. L. Luo, N. L. Wang, and H. Ding, Phys. Rev. Lett. 102, 047003 (2009).

${ }^{43}$ J. Zhao, D.-X. Yao, S. Li, T. Hong, Y. Chen, S. Chang, W. Ratcliff, J. W. Lynn, H. A. Mook, G. F. Chen, J. L. Luo, N. L. Wang, E. W. Carlson, J. Hu, and P. Dai, Phys. Rev. Lett. 101, 167203 (2008).

${ }^{44}$ T. Maier, S. Graser, D. Scalapino, and P. Hirschfeld, arXiv:0903.0008 (unpublished).

${ }^{45}$ H. Q. Yuan, J. Singleton, F. F. Balakirev, S. A. Baily, G. F. Chen, J. L. Luo, and N. L. Wang, Nature (London) 457, 565 (2009).

${ }^{46}$ J. G. Checkelsky, L. Li, G. F. Chen, J. L. Luo, N. L. Wang, and N. P. Ong, arXiv:0811.4668 (unpublished).

${ }^{47}$ R. T. Gordon, C. Martin, H. Kim, N. Ni, M. A. Tanatar, J. Schmalian, I. I. Mazin, S. L. Bud'ko, P. C. Canfield, and R. Prozorov, Phys. Rev. B 79, 100506(R) (2009). 